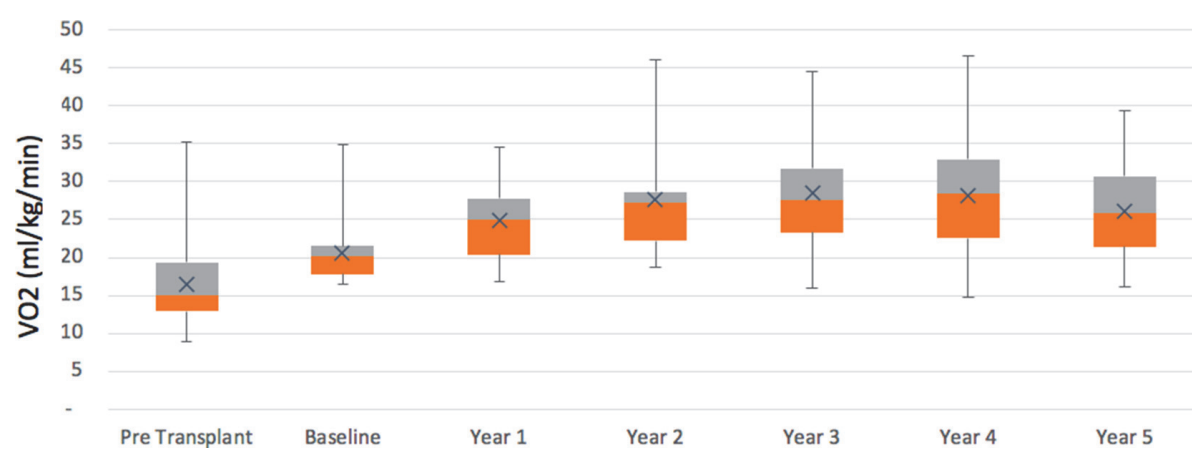

Abstract 129 Figure 1 Box plot representing change in V02 with time post-transplant

Introduction The role of cardiopulmonary exercise testing (CPET) in assessing patients with advanced heart failure (HF) is well established. Exercise capacity is a validated marker of quality of life in this cohort of patients. This study aimed to assess changes in CPET parameters in patients undergoing Heart Transplantation (HTx), subdividing outcomes by underlying aetiology of HF.

Methods A single-centre, retrospective analysis of CPET data including baseline haemodynamic parameters, exercise time (ET), peak oxygen consumption (VO2) and respiratory exchange ratio (RER) of all patients undergoing HTx between July 2011 and December 2018 was conducted. Pre and postHTx CPET parameters were compared and correlation between Pre-HTx CPET parameters and 1-year mortality was analysed.

Results 199 patients underwent HTx, 138 (69.3\%) male, with a median age at transplant of 46 years. Advanced dilated cardiomyopathy (DCM) 62.8\% $(\mathrm{n}=125)$ and ischaemic cardiomyopathy (ICM) $18.6 \%(n=37)$ were the leading indications for HTx. 35 (17.6\%) were bridged to transplant with Ventricular Assist Devices (VAD) .Being unable to perform CPET preHTx was a predictor of 1-year mortality post HTx; RR 1.65 (95\%CI 1.06-2.56; $p=0.024)$. However, when CPET was performed, a low pre-HTx VO2 $(<14 \mathrm{ml} / \mathrm{kg} / \mathrm{min})$ was not a predictor of 1-year mortality; RR 0.93 (95\% CI $0.508-1.715$, $\mathrm{p}=0.824)$.

Pre-HTx and 1 year post-transplant CPET data was available for 77 patients. HTx significantly increased the number of patients achieving greater than $50 \%$ predicted VO2; $86.1 \%$ pre-HTx vs $30.7 \%$ post-HTx $(\mathrm{p}=0.001)$. There was a mean increase in VO2 of $6.94 \mathrm{ml} / \mathrm{kg} / \mathrm{min}$ per patient (95\% CI 5.79 8.08; $\mathrm{p}=0.001)$. When subdivided by underlying HF aetiology, there was no significant difference in the degree of improvement in $\mathrm{VO} 2 ; 58.9 \%$ post-HTx improvement in $\mathrm{VO} 2$ in patients with ICM vs $62.2 \%$ for patients with non-ischaemic DCM $(p=0.24)$. For the subgroup of patients $(n=42)$ who had serial CPETs, the improvements in VO2 were seen within the first 3 years post-HTx, before reaching a plateau after 3 years (figure 1).

Conclusion Patients pre-HTx should be counselled on the objective improvement in exercise capacity seen in this study, as a marker of improved quality of life post-HTx, regardless of the underlying HF aetiology. The inability to perform CPET pre-HTx is associated with higher peri-operative mortality. This study also highlights the importance of CPET to riskstratify patients pre-HTx

Conflict of Interest None

\section{0 \\ HEART FAILURE ADMISSIONS AND DEVICE THERAPY PROVISION DURING THE COVID-19 PANDEMIC}

${ }^{1}$ Rimma Hall, ${ }^{2}$ Rahul Chattopadhyay, ${ }^{3}$ Peter J Pugh. ${ }^{1}$ Cambridge University Hospitals NHS Foundation Trust, Cambridge, UK; ${ }^{2}$ Addenbrooke's Hospital, Cambridge University Hospitals NHS Foundation Trust, ${ }^{3}$ Cambridge University Hospital

\subsection{6/heartjnl-2021-BCS.127}

Introduction The 2020 Covid-19 pandemic saw a marked nationwide reduction in hospital admissions due to cardiovascular disease. In many institutions, including ours, it also saw redistribution of patient care from a specialty-based approach to a ward-based system, meaning patients being admitted and remaining under the care of non-specialist teams. We wished to examine the impact of these changes on heart failure patients presenting during the pandemic and on appropriate delivery of complex device therapies.

Methods The study was undertaken in a large UK teaching hospital, where the annual audit programme includes NICE guidance (Technology Appraisal 314: Implantable Cardioverter Defibrillators and Cardiac Resynchronisation Therapy for Arrhythmias and Heart Failure). As per the NICE guideline, patients admitted to hospital were identified, using discharge codes, with: serious ventricular arrhythmia; familial cardiac condition with high risk of sudden cardiac death (SCD); surgical repair of congenital heart disease; and patients with heart failure and LVEF $<36 \%$. Findings during the pandemic (March to August 2020) were compared with non-pandemic (the same period in 2019). Fisher's exact test was used to compare proportions.

Results Table 1 shows how many patients were eligible for device therapy and how many were offered it. Among nonheart failure patients, device therapy prescription was consistent in the 2 periods observed. Table 2 shows details of heart

Abstract 130 Table 1 Patients admitted to hospital with an indication for device therapy

\begin{tabular}{lllll}
\hline & 2019 & \multicolumn{3}{l}{2020} \\
\hline & Eligible & Offered & Eligible & Offered \\
\hline Serious ventricular arrhythmia & 18 & $16(89 \%)$ & 18 & $16(89 \%)$ \\
Familial cardiac condition with high risk of SCD & 7 & $7(100 \%)$ & 12 & $11(92 \%)$ \\
Surgical repair of congenital heart disease & 1 & $1(100 \%)$ & 1 & $1(100 \%)$ \\
Heart failure and LVEF $<36 \%$ & 49 & $46(94 \%)$ & 42 & $33(79 \%)$ \\
Total* $^{*}$ & 75 & $70(93 \%)$ & 73 & $61(84 \%)$ \\
\hline
\end{tabular}


Abstract 130 Table 2 Heart failure patients admitted to hospital during the study periods

\begin{tabular}{lll}
\hline & 2019 & 2020 \\
\hline Discharge code of HF & 216 & 200 \\
Echo performed & $201(93 \%)$ & $187(93 \%)$ \\
EF $<36 \%$ & $78(36 \%)$ & $80(40 \%)$ \\
Ineligible due to frailty & 12 & 19 \\
Ineligible due to QRS $<120$ ms and NYHA 4 & 14 & 14 \\
Ineligible due to LVEF improvement & 3 & 4 \\
Eligible for device & $49(23 \%$ of HF pts) & $42(21 \%$ of HF pts ) \\
Patients offered device* & $46 / 49(94 \%)$ & $33 / 42(79 \%)$ \\
\hline${ }^{*} \mathrm{P}=0.032019$ v 2020 & &
\end{tabular}

failure admissions during the study periods. Among heart failure patients, an $8 \%$ reduction in hospital admissions due to heart failure was observed during the pandemic. Among these patients, the proportion eligible for device therapy did not alter significantly. However, there was a significant reduction in the proportion of eligible patients who were offered device therapy $(79 \%$ vs $94 \%, p=0.03)$. In addition, there was a trend towards a greater chance of patients being considered too frail for device therapy during the pandemic $(p=0.08)$. Among 12 patients overlooked for device therapy, 10 were not under the care of Cardiology. Among 31 patients considered too frail for device therapy, 26 were not under the care of Cardiology.

Conclusion During the Covid-19 pandemic, a modest reduction in hospital admission for heart failure was observed with a marked fall in identification of those eligible for device therapy. There are a number of possible explanations, including the lack of recognition of indications for device therapy when patients are under the care of non-specialists, an increased perception of patient frailty during a crisis and intense pressure to discharge patients quickly. These findings suggest that cardiac services should actively look for heart failure patients who may have missed out on life-saving device therapies during the pandemic.

Conflict of Interest none

\section{DESIGNING AN EDUCATIONAL APP FOR PATIENTS WITH HEART FAILURE}

${ }^{1}$ Arvind Singhal, ${ }^{2}$ Juhi Tandon, ${ }^{2}$ Tim Ringrose, ${ }^{3}$ Martin Cowie. ${ }^{1}$ Royal Brompton Hospital, London, UK; ${ }^{2}$ Cognitant Group; ${ }^{3}$ mperial College, London

\subsection{6/heartjnl-2021-BCS.128}

Introduction Patient education and engagement is a priority for heart failure (HF) care but given low health literacy and high anxiety around diagnosis in $\mathrm{HF}$, consultations and

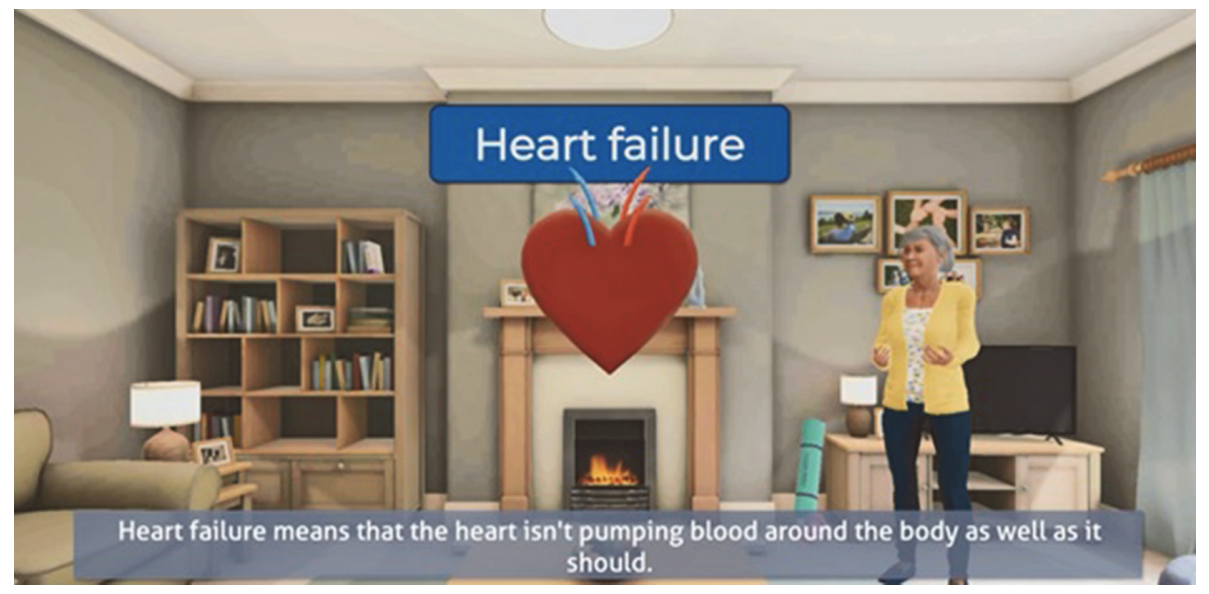

Abstract 131 Figure 1

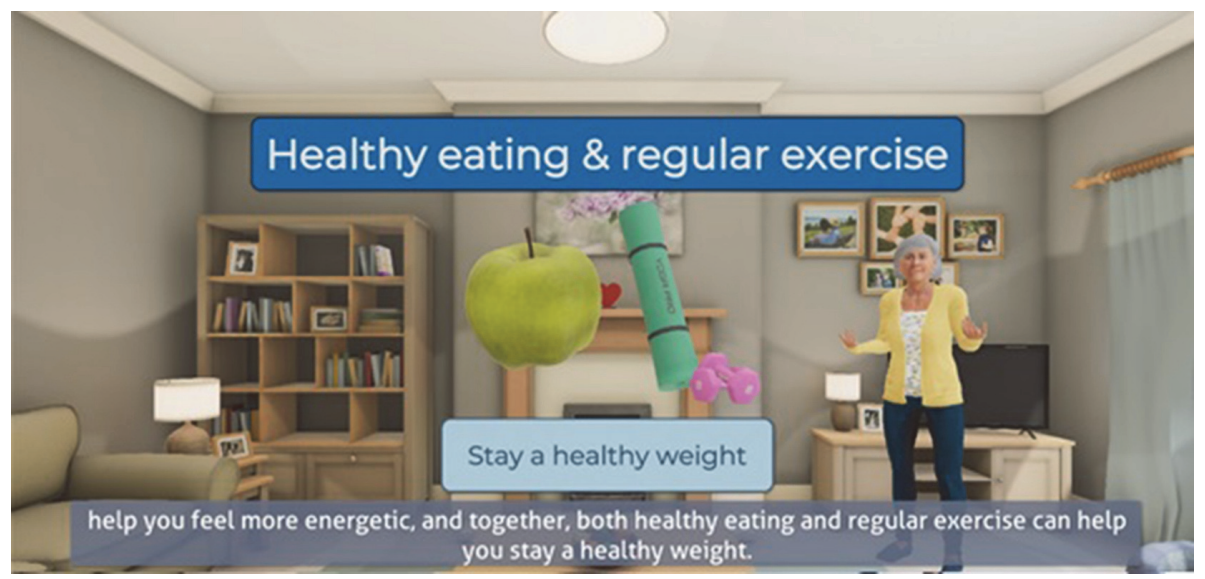

